\title{
Riociguat for the Treatment of Raynaud's Phenomenon: A Single-Dose, Double-Blind, Randomized, Placebo-Controlled Cross-Over Pilot Study (DIGIT)
}

\author{
Michael Huntgeburth ${ }^{1} \cdot$ Johannes Kießling ${ }^{1} \cdot$ Gerrit Weimann $^{2} \cdot$ Verena Wilberg $^{3} \cdot$ Soundos Saleh $^{2}$. \\ Nicolas Hunzelmann ${ }^{4} \cdot$ Stephan Rosenkranz ${ }^{1,5}$
}

Published online: 20 September 2018

(c) The Author(s) 2018

\begin{abstract}
Background and Objective Raynaud's phenomenon (RP) is characterized by transient digital ischemia and is commonly associated with connective tissue disease. Treatment remains unsatisfactory. Here we evaluate the efficacy, safety, and pharmacokinetics of a single dose of the soluble guanylate cyclase stimulator riociguat in RP.

Methods DIGIT was a double-blind, randomized, placebo-controlled pilot study. Patients with primary or secondary RP were randomized to a single oral dose of riociguat $2 \mathrm{mg}$ or placebo in a cross-over design ( $7 \pm 3$ days). Efficacy was assessed as placebo-corrected change in digital blood flow $2 \mathrm{~h}$ post-dose at room temperature (RT) or following cold exposure (CE), measured by laser-speckle contrast analysis. Patients were regarded as responders if placebo-corrected digital blood flow increased by $\geq 10 \%$ from baseline at RT or after CE.

Results Of 20 eligible patients, 17 (85\%) were female and mean [standard deviation (SD)] age was 52 (13.8) years. Placebocorrected changes in digital blood flow were $+46 \%$ [90\% confidence interval (CI) -6 to +98$]$ at RT and - 9\% (90\% CI - 63 to +44$)$ after CE, with high inter-individual variability. Eight patients (40\%) were responders at RT, and $12(60 \%)$ after CE. Riociguat increased mean (SD) digital blood flow in responders at RT by $+136 \%$ (114) and in responders following CE by $+39 \%$ (53). Riociguat was well tolerated, with few adverse events.

Conclusion In this pilot study, single-dose riociguat was well tolerated in patients with RP and resulted in improved digital blood flow in some patient subsets, with high inter-individual variability. Long-term evaluation is warranted.
\end{abstract}

Nicolas Hunzelmann and Stephan Rosenkranz contributed equally to this work

Electronic supplementary material The online version of this article (https://doi.org/10.1007/s40261-018-0698-1) contains supplementary material, which is available to authorized users.

Stephan Rosenkranz

stephan.rosenkranz@uk-koeln.de

1 Clinic III for Internal Medicine, Department of Cardiology, Heart Center, University Hospital of Cologne, Kerpener Str. 62, 50937 Cologne, Germany

2 Bayer AG, Berlin, Germany

3 ClinStat GmbH, Cologne, Germany

4 Department of Dermatology, University of Cologne, Cologne, Germany

5 Heart Center, Cologne Cardiovascular Research Center (CCRC), Cologne, Germany

\section{Key Points}

Single doses of riociguat were well tolerated in patients with Raynaud's phenomenon.

Riociguat was effective in some patient subsets with Raynaud's phenomenon in terms of rapid improvement in digital blood flow at room temperature, with wide inter-individual variation in response.

Riociguat may be a promising new treatment for Raynaud's phenomenon; this warrants further evaluation.

\section{Introduction}

Raynaud's phenomenon (RP) is one of several rheumatologic conditions linked to impaired peripheral circulation $[1,2]$. Characterized as a transient digital ischemia caused 
by vasoconstriction in the digital blood vessels, RP results in a characteristic triphasic color change (pallor, cyanosis, and redness) and often painful symptoms [2-5].

Triggers for acute attacks may be physical, chemical, or emotional [4]; however, cold exposure (CE) or changes in temperature are the most frequent triggers [3, 5-7].

RP may be primary (idiopathic) when occurring in the absence of underlying disease (characterized by normal serum inflammatory markers and nailfold capillaroscopy), or secondary, commonly associated with (but not limited to) connective tissue diseases (CTDs) such as systemic sclerosis $(\mathrm{SSc})[4,5,8]$.

The recommended first-line pharmacotherapy treatments for primary and secondary RP are vasodilators such as dihydropyridine calcium channel blockers (CCBs), angiotensin receptor blockers, or nitrates [9]. Previously, intravenous prostanoids were recommended as second-line therapy, followed by third-line treatment with phosphodiesterase type 5 inhibitors (PDE5i) [9]. More recently, the use of PDE5i as second-line therapy has increased in routine practice based on data from several randomized, controlled trials and a meta-analysis, combined with the falling cost of these drugs $[10,11]$. As a result, the European League Against Rheumatism (EULAR) guidelines for the treatment of SSc recommend PDE5i for patients with severe RP and those who do not respond to CCBs [12]. Despite these treatment options, current medical therapy remains unsatisfactory, with treatments achieving minimal efficacy $[9,13]$. Secondary RP is particularly difficult to treat and current evidence on the efficacy of RP treatment in patients with SSc is inconclusive, highlighting the need for further research into new treatment options [14].

An important aspect of the pathophysiology of RP is endothelial dysfunction, which is associated with reduced nitric oxide (NO) bioavailability in the peripheral vasculature, leading to decreased levels of cyclic guanosine monophosphate (cGMP) and vasoconstriction [4]. Therefore, the NO-soluble guanylate cyclase (sGC)-cGMP signaling pathway represents an attractive target for the treatment of RP. Indeed, PDE5i, which promote vasodilatation by increasing intracellular cGMP levels, were shown to improve blood flow and symptoms in patients with primary and secondary RP [15-17]. However, over time, RP is associated with progressive endothelial dysfunction and declining levels of endogenous NO; therefore, enhancing cGMP production independent of NO by directly stimulating sGC may be a more effective approach.

Riociguat is a first-in-class sGC stimulator and has been developed as an effective treatment for various forms of pulmonary hypertension. It has a dual mode of action: (1) direct stimulation of sGC, independent from NO, and (2) sensitization of sGC to endogenous NO by stabilizing NO-sGC binding. As a result, riociguat efficiently stimulates the
NO-sGC-cGMP pathway and leads to increased intracellular levels of cGMP and, in contrast to PDE5i, its action is not dependent on endogenous NO levels [18-20].

Here we present the results of the pilot DIGIT study (DIGital flow enhancement in Raynaud's disease with an sGCstImulaTor) designed to investigate the safety, efficacy, and pharmacokinetics of a single dose of riociguat in patients with RP.

\section{Methods}

\subsection{Study Design and Population}

DIGIT was a single-dose, double-blind, randomized, placebo-controlled, cross-over, exploratory study (Supplementary Fig. 1). Patients aged 18-70 years were eligible for inclusion if they had primary RP or secondary RP associated with limited cutaneous SSc (lcSSc), diffuse cutaneous SSc (dcSSc), undifferentiated SSc, or SSc overlap syndrome for $\geq 1$ year prior to screening. Exclusion criteria consisted of co-medication with vasodilators including PDE5i, endothelin receptor antagonists, CCBs, prostacyclin analogs, or nitrates. All vasodilator medications had to be discontinued at least 1 week prior to enrollment until $24 \mathrm{~h}$ after last study drug intake. Other exclusion criteria were: systolic blood pressure $<105$ or $>160 \mathrm{mmHg}$ at rest; diastolic blood pressure $<50$ or $>95 \mathrm{mmHg}$ at rest; any condition that may have interfered with normal pharmacokinetics of riociguat; smoking; abnormal electrocardiographic or heart rate findings; or clinically relevant findings on physical examination.

\subsection{Study Procedures}

Eligible patients were randomized to receive a single oral dose of either riociguat (Adempas ${ }^{\circledR}$, Bayer AG, Berlin, Germany) $2 \mathrm{mg}$ or matching placebo, with cross-over separated by $7 \pm 3$ days before receiving the other respective study drug. Some studies of PDE5i have reported prolonged effects on vascular function, which exceed their plasma half-life. The separation period ensured adequate washout and allowed time for any long-lasting vascular effects of riociguat to fade between treatments [15, 17, 21].

Perfusion of the right index finger was measured by laserspeckle contrast analysis (PeriCam PSI Systems, Perimed, Järfälla, Sweden) at baseline and $2 \mathrm{~h}$ post-dose at room temperature (RT) $\left(20-24{ }^{\circ} \mathrm{C}\right.$ for $\left.\geq 20 \mathrm{~min}\right)$. Perfusion was also measured immediately after cold water exposure (CE) (both hands at $12{ }^{\circ} \mathrm{C}$ for $5 \mathrm{~min}$ ) at baseline and at $2 \mathrm{~h}$ post-dose. After CE, the average of the 5 different time points (mean of 2 measurements each at $0,3,5,7$, and $15 \mathrm{~min}$ ) of the distal phalanx of the right index finger was calculated. In the case of a contraindication to using the right index finger 
(2 patients), measurements were performed on the left index finger. At $2 \mathrm{~h}$ post-dose, venous blood samples were collected, preferably from the cubital fossa of the left arm, for the measurement of riociguat plasma concentration at the time of digital blood flow measurement.

\subsection{Efficacy and Safety Measures}

The efficacy of riociguat was assessed using placebo-corrected change from baseline in digital blood flow $2 \mathrm{~h}$ postdose at RT and following CE. The pre-specified responder criterion was a placebo-corrected increase in digital blood flow by $\geq 10 \%$ versus baseline at $2 \mathrm{~h}$ post-dose at RT or after $\mathrm{CE}$. The responder cut-off was based on a previous study in patients with RP, which showed a digital blood flow increase of approximately $10 \% 1 \mathrm{~h}$ after a single dose of the PDE5i vardenafil at RT or after CE [17]. Adverse events (AEs) were recorded for at least 7 days, with active monitoring for serious AEs (SAEs) for a further 23 days. All AEs were assessed and documented by the investigator in terms of seriousness, intensity (mild, moderate, or severe), and causal relationship to administration of treatment and protocol-required procedure(s). SAEs were defined as any AEs that resulted in death or were life threatening, or that required inpatient hospitalization or prolongation of hospitalization. An AE was also considered serious if it resulted in persistent or significant disability/incapacity (disability meaning a substantial disruption of the patient's ability to conduct normal life functions); if it was a congenital abnormality or birth defect; or if it was another medically important serious event as judged by the investigator. Blood and urine samples were collected at screening and follow-up for determination of hematologic parameters (leukocytes, erythrocytes, hemoglobin, hematocrit, and platelets) and clinical chemistry (aspartate aminotransferase, alanine aminotransferase, lactate dehydrogenase, creatine kinase, urea, sodium, and potassium).

\subsection{Bioanalytic Methods}

Riociguat concentration was determined in plasma after protein precipitation with methanol containing an internal standard followed by separation using high-pressure liquid chromatography and tandem mass spectrometric detection. The calibration range was 2.00 [lower limit of quantification (LLOQ)] to $500 \mu \mathrm{g} / \mathrm{L}$ (upper limit of quantification). Mean inter-assay accuracy of back-calculated concentrations (except LLOQ) in calibrators was $96.8-104 \%$ and precision $\leq 8.63 \%$. Accuracy and precision at the LLOQ were 99.8 and $4.63 \%$, respectively. Quality control samples in the concentration range $6.00-400 \mu \mathrm{g} / \mathrm{L}$ were determined with an accuracy of $99-102 \%$ and a precision of $2.74-5.59 \%$. All samples were stored at or below $-15^{\circ} \mathrm{C}$ and analyzed within 9 months after sampling.

\subsection{Statistical Analysis}

Based on previous experience [17], the chosen sample size of 20 valid patients measured in a cross-over design was considered sufficient to fulfill the objectives of the study. No formal statistical sample size estimation was performed in this exploratory study. The number of digital blood flow responders at $\mathrm{RT}$ and after $\mathrm{CE}$, including subgroup analyses according to medical grouping, was calculated. Summary statistics for digital blood flow and percentage change from baseline were calculated by response. The percentage change from baseline of digital blood flow was analyzed by an explorative analysis of variance. Point estimates and 2 -sided $90 \%$ confidence intervals (CIs) for the difference "riociguat-placebo" were calculated. Individual subject profile plots for placebo-corrected change in digital blood flow were used for visualization.

\section{Results}

\subsection{Patients}

Of the 23 randomized patients, 20 completed the study without major protocol deviations and entered the pharmacodynamic analysis group (Supplementary Fig. 2). All 23 patients who received study drug were included in the safety analysis group, and 22 were included in the pharmacokinetic analysis group. The baseline characteristics of the 20 patients who completed the study without major protocol deviations are presented in Table 1. Most patients $(65 \%)$ were treatment naïve, and of the previously treated patients $(n=7), 5$ had received CCBs, which were discontinued at least 1 week prior to enrollment.

\subsection{Effects of Single-Dose Riociguat on Digital Perfusion}

In the 20 patients who completed the study, the placebocorrected change in digital blood flow at RT 2 h postdose was $+46 \%(90 \%$ CI -6 to +98$)$ (Fig. 1a; Table 2), as riociguat increased mean [standard deviation (SD)] digital blood flow by $+41 \%$ (109) compared with $-5 \%$ (59) after placebo. Following CE, the placebo-corrected change from baseline in digital blood flow at $2 \mathrm{~h}$ postdose was $-9 \%(90 \% \mathrm{CI}-63$ to +44$)$ (i.e. digital perfusion was higher with placebo) (Fig. 1b; Table 2), as mean (SD) digital blood flow increased by $+15 \%$ (51) with riociguat and by $+25 \%$ (114) with placebo. Variability in digital blood flow between subjects was high following both single doses of riociguat and placebo; individual placebo-corrected changes from baseline in digital blood flow following treatment with riociguat at RT and after 
Table 1 Baseline characteristics ${ }^{\mathrm{a}}$

Placebo-riociguat $(n=11)$

Riociguat-placebo $(n=9)$

Total $(N=20)$

Sex, $n(\%)$

Male

$1(9.1)$

$10(90.9)$

$2(22.2)$

$3(15.0)$

Female

$11(100.0)$

$7(77.8)$

17 (85.0)

Race, $n(\%)$

Caucasian

$52.0(15.8)$

$9(100.0)$

$20(100.0)$

Mean (SD) age, years

$52.0(11.8)$

$52.0(13.8)$

Smoking history, $n(\%)$

Former

$6(54.5)$

$1(11.1)$

$7(35.0)$

Never

$5(45.5)$

8 (88.9)

$13(65.0)$

Alcohol use, $n(\%)$

Abstinent

7 (63.6)

$8(88.9)$

$15(75.0)$

Light

$4(36.4)$

$1(11.1)$

$5(25.0)$

Prior medication, $n(\%)$

Amlodipine

$1(9.1)$

$0(0.0)$

$0(0.0)$

Diltiazem

$1(9.1)$

Iloprost

$2(18.2)$

Nifedipine

0

Angiotensin receptor blockers

PDE5 inhibitor

0

$0(0.0)$

$1(5.0)$

$1(11.1) \quad 1(5.0)$

$1(11.1) \quad 1(5.0)$

$0(0.0) \quad 1(5.0)$

$1(11.1) \quad 3(15.0)$

RP etiology, $n(\%)$

Primary (idiopathic)

Secondary to dcSSc

$1(9.1)$

0

0

0

0

Secondary to lcSSc

$5(45.5)$

$3(27.3)$

$2(18.2)$

$2(22.2)$

$3(15.0)$

5 (55.6)

$10(50.0)$

$2(22.2)$

$5(25.0)$

Secondary to SSc overlap

$0(0.0)$

$2(10.0)$

$d c S S c$ diffuse cutaneous systemic sclerosis, $l c S S c$ limited cutaneous systemic sclerosis, $P D E 5$ phosphodiesterase-5, $R P$ Raynaud's phenomenon, $S D$ standard deviation, SSc systemic sclerosis

${ }^{a}$ Includes all patients who completed the study

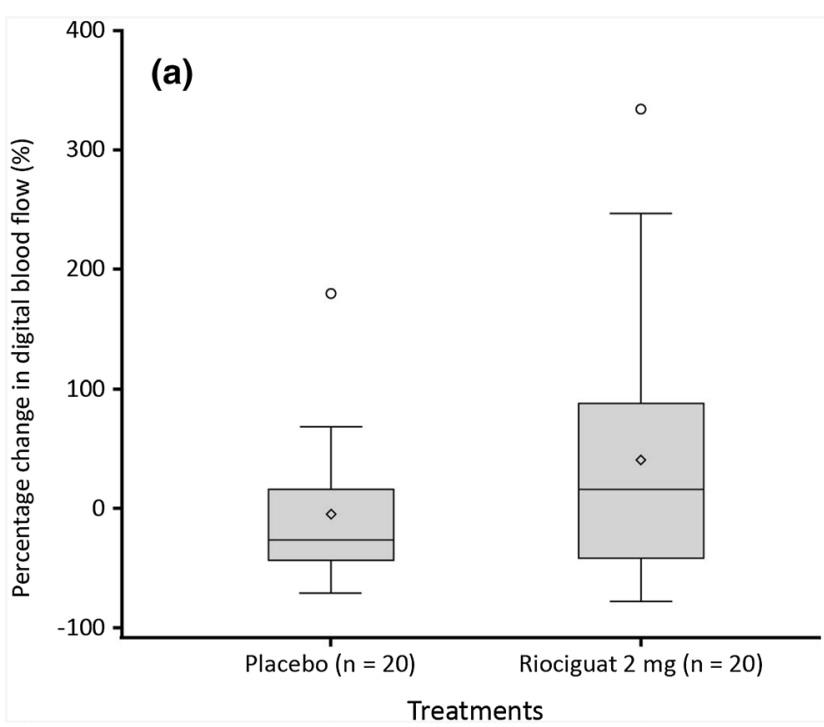

Fig. 1 Box plot for percentage change from baseline in digital blood flow at room temperature (a) and following cold exposure (b) $(N=20)$. Box: 25 th to 75 th percentile; horizontal line: median; dia- (b)

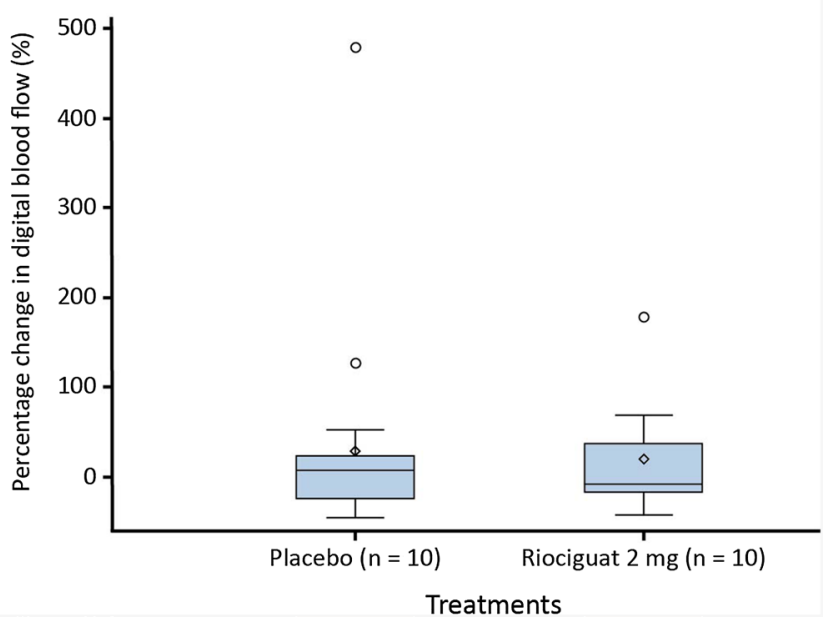

mond: arithmetic mean; vertical lines extend from the box to a maximum distance of 1.5 interquartile ranges; outliers are plotted separately (circles) 
Table 2 Percentage change from baseline in digital blood flow (PU) in overall population $(N=20)$ and responders $(n=12)^{\mathrm{a}}$ at room temperature and immediately after cold exposure

\begin{tabular}{|c|c|c|c|c|c|}
\hline \multirow[t]{2}{*}{ Treatment group } & \multirow[t]{2}{*}{ Time point } & \multicolumn{2}{|c|}{ Value at time } & \multicolumn{2}{|c|}{$\%$ Change from baseline } \\
\hline & & Mean (SD) & Range & Mean (SD) & Range \\
\hline \multicolumn{6}{|c|}{ Overall population $(N=20)$} \\
\hline \multicolumn{6}{|l|}{ Room temperature } \\
\hline \multirow[t]{2}{*}{ Riociguat $2 \mathrm{mg}$} & $0 \mathrm{~min}$ & $53.6(30.7)$ & $18.0-124.0$ & & \\
\hline & $2 \mathrm{~h}$ post-dose & $62.0(47.7)$ & $14.0-204.0$ & $40.6(109.2)$ & -78.2 to 334.0 \\
\hline \multirow[t]{2}{*}{ Placebo } & $0 \mathrm{~min}$ & $69.5(38.9)$ & $22.0-173.0$ & & \\
\hline & $2 \mathrm{~h}$ post-dose & $58.2(39.7)$ & $13.0-199.0$ & $-4.7(58.8)$ & -71.1 to 180.6 \\
\hline \multicolumn{6}{|c|}{ Following cold exposure } \\
\hline \multirow[t]{2}{*}{ Riociguat $2 \mathrm{mg}$} & $0 \min$ & $31.8(24.0)$ & $9.0-99.0$ & & \\
\hline & $2 \mathrm{~h}$ post-dose & $32.7(21.2)$ & $8.0-91.0$ & $15.1(51.0)$ & -42.3 to 179.2 \\
\hline \multirow[t]{2}{*}{ Placebo } & $0 \mathrm{~min}$ & $30.6(16.0)$ & $12.0-67.0$ & & \\
\hline & $2 \mathrm{~h}$ post-dose & $33.5(23.9)$ & $12.0-98.0$ & $24.7(114.0)$ & -45.2 to 480.0 \\
\hline \multicolumn{6}{|l|}{ Responders $(n=12)$} \\
\hline \multicolumn{6}{|l|}{ Room temperature } \\
\hline \multirow[t]{2}{*}{ Riociguat $2 \mathrm{mg}$} & $0 \min$ & $44.4(34.7)$ & $18.0-124.0$ & & \\
\hline & $2 \mathrm{~h}$ post-dose & $92.1(58.7)$ & $30.0-204.0$ & $135.7(113.8)$ & -15.3 to 334.0 \\
\hline \multirow[t]{2}{*}{ Placebo } & $0 \mathrm{~min}$ & $85.3(43.9)$ & $34.0-173.0$ & & \\
\hline & $2 \mathrm{~h}$ post-dose & $51.9(16.2)$ & $26.0-84.0$ & $-27.2(38.5)$ & -69.4 to 47.1 \\
\hline \multicolumn{6}{|c|}{ Following cold exposure } \\
\hline \multirow[t]{2}{*}{ Riociguat $2 \mathrm{mg}$} & $0 \min$ & $29.6(25.2)$ & $13.0-99.0$ & & \\
\hline & $2 \mathrm{~h}$ post-dose & $37.0(24.4)$ & $17.0-91.0$ & $38.9(53.3)$ & -9.1 to 179.0 \\
\hline \multirow[t]{2}{*}{ Placebo } & $0 \mathrm{~min}$ & $32.3(17.3)$ & $12.0-67.0$ & & \\
\hline & $2 \mathrm{~h}$ post-dose & $25.9(11.6)$ & $13.0-49.0$ & $-13.9(22.2)$ & -45.2 to 19.0 \\
\hline
\end{tabular}

$P U$ perfusion units, $S D$ standard deviation

${ }^{a}$ Responders were defined as patients with a placebo-corrected digital blood flow increase $\geq 10 \%$ from baseline $2 \mathrm{~h}$ post-drug intake at room temperature or immediately after cold exposure

$\mathrm{CE}$ are shown in Fig. 2. Figure 3 shows laser speckle contrast analysis images after dosing with riociguat (a) or placebo (b).

A total of 8 patients (40\%) were classified as responders to riociguat at RT, and 12 patients $(60 \%)$ as responders after $\mathrm{CE}$, according to the predefined criterion. In responders, riociguat led to a mean (SD) increase from baseline in digital blood flow of $+136 \%$ (114) at RT (Table 2) and $+39 \%$ (53) after CE (Table 2). The highest response rates following $\mathrm{CE}$ were seen in patients with $\operatorname{lcSSc}(80 \% ; n=4)$ and primary RP $(67 \% ; n=2)$ (Fig. 4).

\subsection{Pharmacokinetics}

The geometric mean (SD) concentration of riociguat in plasma $2 \mathrm{~h}$ post-dose was $76(1.5) \mu \mathrm{g} / \mathrm{L}$ (range $35-121 \mu \mathrm{g} / \mathrm{L}$ ). Plasma concentration of riociguat $2 \mathrm{~h}$ post-dose was comparable in responders [geometric mean (SD) $81(1.5) \mu \mathrm{g} / \mathrm{L}$; range 35-121] and non-responders [geometric mean (SD) $71(1.6) \mu \mathrm{g} / \mathrm{L}$; range 38-117].

\subsection{Safety}

All patients who received a dose of riociguat were included in the safety analysis set $(n=23)$. AEs were reported in 6 patients while receiving riociguat (headache, $n=5$; and dyspepsia, $n=1$ ) and in 2 patients while receiving placebo (malaise, $n=1$; and pain in extremity, $n=1$ ). One patient discontinued study drug and withdrew consent after the first treatment period due to side effects; after unblinding, this patient's treatment was shown to be placebo.

No SAEs were reported, and no clinically relevant hematologic or biochemical findings were observed. Changes in heart rate and blood pressure were minimal throughout the treatment period, with no patient experiencing symptomatic hypotension (Supplementary Table 1). 
Fig. 2 Individual placebocorrected percentage change from baseline in digital blood flow at $2 \mathrm{~h}$ post-administration of riociguat $2 \mathrm{mg}$ at room temperature (a) and following cold exposure $(\mathbf{b})(N=20)$. Red dashed line $=$ reference line for a placebo-corrected change of $+10 \%$
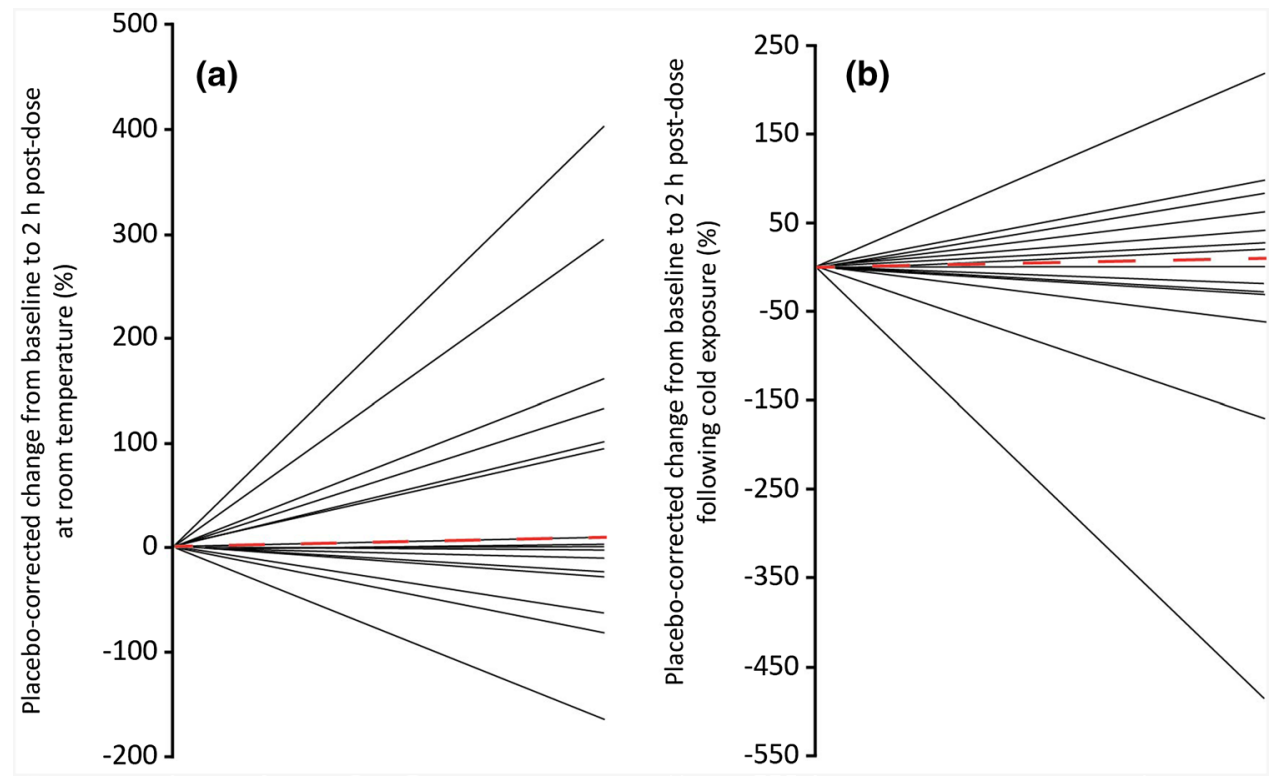

(a) Riociguat $2 \mathrm{mg}$

Fig. 3 Laser speckle contrast analysis images of the distal phalanx of the right index finger of a patient 7 min after cold exposure following dosing with riociguat (a) and placebo (b)

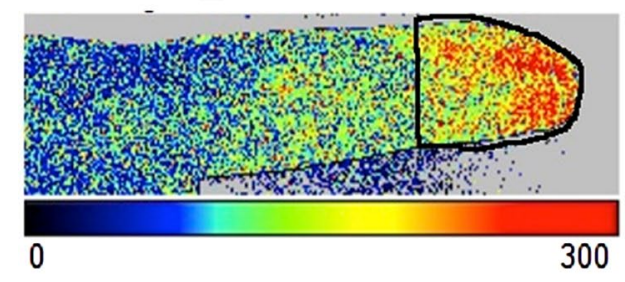

(b) Placebo

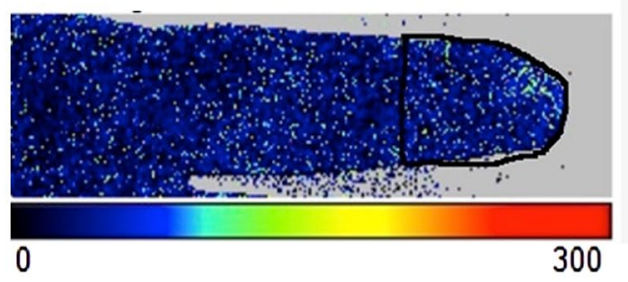

Blood collection was performed without difficulties. No patient reported anxiety or pain during collection, and no clinical sign of a vasovagal reaction was observed.

\section{Discussion}

In this pilot study, a single dose of riociguat improved digital perfusion in some patient subsets with RP. Perfusion increased by $\geq 10 \%$ in $40 \%$ of patients at RT and $60 \%$ following CE. Although patient numbers were small, we observed considerable variability in the response rates of patients with different forms of RP. However, responses were observed across the subgroups, including in difficult-totreat secondary RP due to dcSSc or SSc overlap syndrome. Improved blood flow was seen particularly at RT, where the placebo-corrected change in digital perfusion was $+46 \%$ in all patients and $+136 \%$ in "responders", whereas only modest changes were seen after $\mathrm{CE}$, and the mean change with riociguat was numerically smaller than that seen with placebo. This may be expected, as RT represents a much more physiologic and realistic situation when compared with an extreme induction of RP in ice-cold water, which is expected to be much more difficult to overcome. It is also possible that the CE test may have resulted in cold-induced vasodilation in some participants. The measurement of blood flow at 5 time points, to obtain an average representative value, will have reduced the impact of cold-induced vasodilation (if present) on the results. In patients who responded to riociguat we observed less decrease in blood flow after cold exposure (Table 2). In view of the absence of pain, anxiety or a vasovagal reaction, it seems unlikely that the procedure interfered with blood flow distal to the puncture site.

The absence of a response to riociguat in some patients may be a result of them receiving only a single $2 \mathrm{mg}$ dose. Single doses of riociguat produce hemodynamic effects in healthy subjects [22] and patients with pulmonary hypertension [23] or heart failure [24], but chronic dosing may be necessary to obtain a response in heavily diseased digital blood vessels. A prospective analysis of the PATENT-1 and PATENT-2 studies of riociguat in pulmonary arterial hypertension $(\mathrm{PAH})$ found that the hemodynamic effects of riociguat were less marked in the subgroup of patients with PAH associated with CTD than in the overall PATENT 
Fig. 4 Proportions of responders and non-responders to riociguat after cold exposure in all patients and by etiology of RP. Responders were defined as patients with a placebocorrected digital blood flow increase $\geq 10 \%$ from baseline $2 \mathrm{~h}$ post-drug intake at room temperature or following cold exposure. $R P$ Raynaud's phenomenon, $S D$ standard deviation, $S S c$ systemic sclerosis

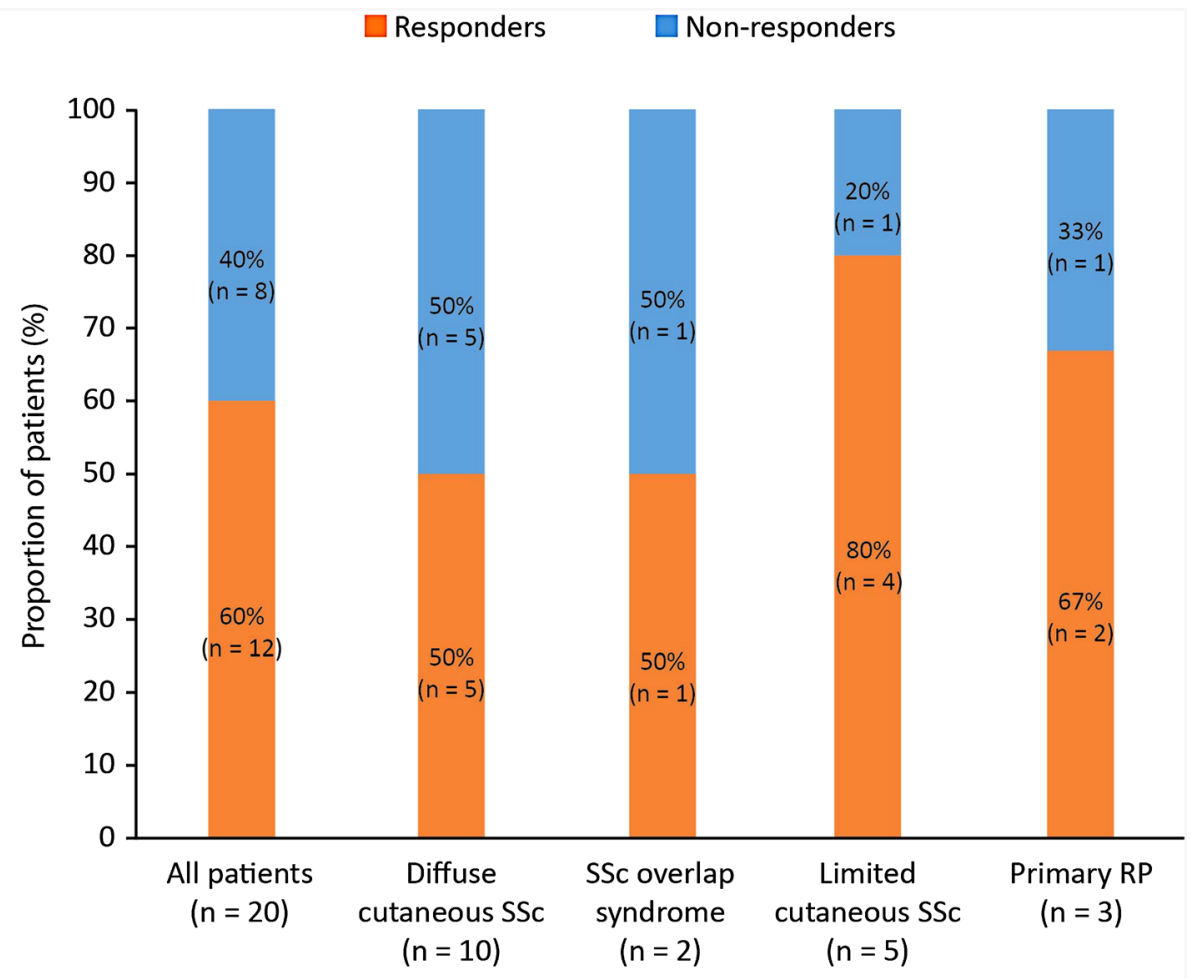

Riociguat mean concentration in plasma $2 \mathrm{~h}$ 76 73 107

88

55

post-dose, $\mu \mathrm{g} / \mathrm{L}$

Geometric SD
2
1
1 population. This result suggests that vessels of patients with CTD may be relatively resistant to treatment.

In previous studies, the effects of PDE5 inhibition on endothelial function and clinical symptoms persisted for at least 2 weeks following discontinuation of the study drug, i.e. exceeded the plasma half-life of the drug [15, 17]. This may suggest that the molecular mechanisms affecting endothelial function are altered by chronic inhibition of PDE5 beyond the acute functional vasoactive effects. The NO-independent action of sGC stimulators distinguishes riociguat from PDE5i, which require NO to function properly. Therefore, the efficacy of PDE5i may be limited in the presence of low endogenous NO levels, which are known to be depleted in RP [25, 26]. In contrast to PDE5i, riociguat targets the NO-sGC-cGMP pathway via a dual mode of action that leads to increased sensitivity of sGC to NO and increased sGC activity independent of NO [19, 23]. This results in potent vasodilatory effects as well as potentially disease-modifying properties such as anti-fibrotic, anti-proliferative, and anti-inflammatory activity [20]. These effects could be expected to confer benefits in RP beyond simple vasodilation but take time to develop and would not be expected to occur with a single dose as administered in the current study.

Riociguat was well tolerated in this study. Seven patients experienced AEs that were considered to be study drug related, and AEs were consistent with those observed in previous studies [22, 23, 27-31]. There were no SAEs or deaths. One patient discontinued the study after the first phase due to subjective side effects of the study drug, which after unblinding proved to be placebo. Therefore, the discontinuation is not regarded as study drug related. The dose of riociguat used in DIGIT ( $2 \mathrm{mg}$ ) was within the range of that used in patients with pulmonary hypertension (range 1-2.5 mg 3 times daily) [30, 31], and the mean plasma concentrations of riociguat at $2 \mathrm{~h}$ post-dose (i.e. when digital blood flow was measured) were in the range of concentrations previously observed in healthy subjects and patients with pulmonary hypertension following single dosing [22, 23].

Several limitations should be allowed for when considering the findings of this study. The small, heterogeneous population precluded a robust evaluation of the responder and RP subgroups, and the findings should therefore be 
interpreted with caution. Although measurement of digital blood flow $2 \mathrm{~h}$ post-administration demonstrated the potential of riociguat for providing immediate improvement, limiting the study to a single time point prevented evaluation of the long-term efficacy, and the cumulative effect on symptoms could not be assessed. Finally, the high interindividual variability of blood flow observed in this study may also limit interpretation of the results. This again may be due in part to the small, heterogeneous population; however, differing response to treatment due to physiologic or other factors should not be excluded and may warrant further investigation.

Although it has limitations, the findings from this exploratory study are encouraging and pave the way for further studies evaluating the effects of riociguat on blood flow and symptoms (e.g. the Raynaud Condition Score) following longer exposure to treatment, to establish the potential role of riociguat for the treatment of RP.

\section{Conclusion}

In this pilot study, a single dose of riociguat was well tolerated and resulted in rapid improvement in digital blood flow, particularly at RT, in some patient subsets with primary and secondary RP, with a high rate of responders following CE. Overall, our results suggest for the first time that riociguat may be a promising new treatment option for RP, and evaluation of long-term effects on blood flow and clinical symptoms is warranted.

Acknowledgements This study contains parts of a doctoral thesis by JK.

Author Contributions All authors contributed to the conception and design of the study, the collection and interpretation of the data, and the drafting and revising of the manuscript, and approved the final version for publication.

\section{Compliance with Ethical Standards}

Conflict of interest $\mathrm{MH}$ and $\mathrm{JK}$ report no conflicts. NH received a research grant and honoraria for lectures from Bayer AG. SR received honoraria for lectures and/or consultancy from Bayer AG and MSD, and his institution received research grants from Bayer AG. GW and SS are employees of Bayer AG. VW is an employee of ClinStat GmbH.

Ethical approval Protocol and all protocol amendments were reviewed and approved by the Ethics Committee of the University Hospital of Cologne (Ethikkommission der Medizinischen Fakultaet, Kerpener Str. 62, 50937 Cologne) before the start of the study, ethics approval number 13-174. As applicable according to local regulations, the protocol was reviewed and approved by the BfArM (Bundesinstitut fuer Arzneimittel und Medizinprodukte, Kurt-GeorgKiesinger-Allee 3, 53175 Bonn, Germany) on 29 July 2013. The study was conducted in accordance with the Declaration of Helsinki, and Good Clinical Practice.
Informed consent All patients gave written informed consent before entering the study.

Funding This study was supported by Bayer AG (Berlin, Germany). Verena Wilberg was assigned by Bayer AG (Berlin, Germany) to conduct the analyses. Medical writing assistance was provided by Adelphi Communications Limited (Bollington, UK), funded by Bayer AG (Berlin, Germany).

Open Access This article is distributed under the terms of the Creative Commons Attribution-NonCommercial 4.0 International License (http://creativecommons.org/licenses/by-nc/4.0/), which permits any noncommercial use, distribution, and reproduction in any medium, provided you give appropriate credit to the original author(s) and the source, provide a link to the Creative Commons license, and indicate if changes were made.

\section{References}

1. Isenberg DA, Black C. ABC of rheumatology. Raynaud's phenomenon, scleroderma, and overlap syndromes. BMJ. 1995;310(6982):795-8.

2. Pope JE. The diagnosis and treatment of Raynaud's phenomenon: a practical approach. Drugs. 2007;67(4):517-25.

3. Wigley FM, Flavahan NA. Raynaud's phenomenon. N Engl J Med. 2016;375(6):556-65.

4. Prete M, Fatone MC, Favoino E, Perosa F. Raynaud's phenomenon: from molecular pathogenesis to therapy. Autoimmun Rev. 2014;13(6):655-67.

5. Wigley FM. Clinical practice. Raynaud's phenomenon. N Engl J Med. 2002;347(13):1001-8.

6. Maverakis E, Patel F, Kronenberg DG, Chung L, Fiorentino D, Allanore Y, Guiducci S, Hesselstrand R, Hummers LK, Duong C, Kahaleh B, Macgregor A, Matucci-Cerinic M, Wollheim FA, Mayes MD, Gershwin ME. International consensus criteria for the diagnosis of Raynaud's phenomenon. J Autoimmun. 2014;48-49:60-5.

7. Goundry B, Bell L, Langtree M, Moorthy A. Diagnosis and management of Raynaud's phenomenon. BMJ. 2012;344:e289.

8. Levien TL. Advances in the treatment of Raynaud's phenomenon. Vasc Health Risk Manag. 2010;6:167-77.

9. Hughes M, Ong VH, Anderson ME, Hall F, Moinzadeh P, Griffiths B, Baildam E, Denton CP, Herrick AL. Consensus best practice pathway of the UK Scleroderma Study Group: digital vasculopathy in systemic sclerosis. Rheumatology (Oxford). 2015;54(11):2015-24.

10. Denton CP, Hughes M, Gak N, Vila J, Buch MH, Chakravarty K, Fligelstone K, Gompels LL, Griffiths B, Herrick AL, Pang J, Parker L, Redmond A, van Laar J, Warburton L, Ong VH, Bsr, Bhpr Standards G, Audit Working G. BSR and BHPR guideline for the treatment of systemic sclerosis. Rheumatology (Oxford). 2016;55(10):1906-10. https://doi.org/10.1093/rheumatology/ kew224.

11. Herrick AL. Recent advances in the pathogenesis and management of Raynaud's phenomenon and digital ulcers. Curr Opin Rheumatol. 2016;28(6):577-85. https://doi.org/10.1097/BOR.0000000000 000332 .

12. Kowal-Bielecka O, Fransen J, Avouac J, Becker M, Kulak A, Allanore Y, Distler O, Clements P, Cutolo M, Czirjak L, Damjanov N, del Galdo F, Denton CP, JrHW Distler, Foeldvari I, Figelstone K, Frerix M, Furst DE, Guiducci S, Hunzelmann N, Khanna D, Matucci-Cerinic M, Herrick AL, van den Hoogen F, 
van Laar JM, Riemekasten G, Silver R, Smith V, Sulli A, Tarner I, Tyndall A, Welling J, Wigley F, Valentini G, Walker UA, Zulian F, Miller-Ladner U. Update of EULAR recommendations for the treatment of systemic sclerosis. Ann Rheum Dis. 2017. https:// doi.org/10.1136/annrheumdis-2016-20990.

13. Ennis H, Anderson ME, Wilkinson J, Herrick AL (2014) Calcium channel blockers for primary Raynaud's phenomenon. Cochrane Database Syst Rev 1:CD002069.

14. Garcia de la Pena L, Nishishinya MB, Pereda CA, Loza E, Sifuentes Giraldo WA, Roman Ivorra JA, Carreira P, RuaFigueroa I, Pego-Reigosa JM, Munoz-Fernandez S. Efficacy of Raynaud's phenomenon and digital ulcer pharmacological treatment in systemic sclerosis patients: a systematic literature review. Rheumatol Int. 2015;35(9):1447-59.

15. Fries R, Shariat K, von Wilmowsky H, Bohm M. Sildenafil in the treatment of Raynaud's phenomenon resistant to vasodilatory therapy. Circulation. 2005;112(19):2980-5. https://doi. org/10.1161/CIRCULATIONAHA.104.523324.

16. Caglayan E, Huntgeburth M, Karasch T, Weihrauch J, Hunzelmann N, Krieg T, Erdmann E, Rosenkranz S. Phosphodiesterase type 5 inhibition is a novel therapeutic option in Raynaud disease. Arch Intern Med. 2006;166(2):231-3.

17. Caglayan E, Axmann S, Hellmich M, Moinzadeh P, Rosenkranz $S$. Vardenafil for the treatment of raynaud phenomenon: a randomized, double-blind, placebo-controlled crossover study. Arch Intern Med. 2012;172(15):1182-4.

18. Stasch JP, Hobbs AJ. NO-independent, haem-dependent soluble guanylate cyclase stimulators. Handb Exp Pharmacol. 2009;191:277-308. https://doi.org/10.1007/978-3-540-68964 $-5 \_13$.

19. Stasch JP, Pacher P, Evgenov OV. Soluble guanylate cyclase as an emerging therapeutic target in cardiopulmonary disease. Circulation. 2011;123(20):2263-73.

20. Stasch JP, Evgenov OV. Soluble guanylate cyclase stimulators in pulmonary hypertension. Handb Exp Pharmacol. 2013;218:279-313.

21. Desouza C, Parulkar A, Lumpkin D, Akers D, Fonseca VA. Acute and prolonged effects of sildenafil on brachial artery flow-mediated dilatation in type 2 diabetes. Diabetes Care. 2002;25(8):1336-9.

22. Frey R, Mück W, Unger S, Artmeier-Brandt U, Weimann G, Wensing G. Single-dose pharmacokinetics, pharmacodynamics, tolerability, and safety of the soluble guanylate cyclase stimulator BAY 63-2521: an ascending-dose study in healthy male volunteers. J Clin Pharmacol. 2008;48(8):926-34.
23. Grimminger F, Weimann G, Frey R, Voswinckel R, Thamm M, Bolkow D, Weissmann N, Mück W, Unger S, Wensing G, Schermuly RT, Ghofrani HA. First acute haemodynamic study of soluble guanylate cyclase stimulator riociguat in pulmonary hypertension. Eur Respir J. 2009;33(4):785-92.

24. Bonderman D, Pretsch I, Steringer-Mascherbauer R, Jansa P, Rosenkranz S, Tufaro C, Bojic A, Lam CS, Frey R, Ochan KM, Unger S, Roessig L, Lang IM. Acute hemodynamic effects of riociguat in patients with pulmonary hypertension associated with diastolic heart failure (DILATE-1): a randomized, double-blind, placebo-controlled, single-dose study. Chest. 2014;146(5):1274-85.

25. Baumhakel M, Bohm M. Recent achievements in the management of Raynaud's phenomenon. Vasc Health Risk Manag. 2010;6:207-14.

26. Herrick AL. Pathogenesis of Raynaud's phenomenon. Rheumatology (Oxford). 2005;44(5):587-96.

27. Ghofrani HA, Galiè N, Grimminger F, Humbert M, Keogh AM, Langleben D, Ochan Kilama M, Neuser D, Rubin LJ (2012) Riociguat for the treatment of pulmonary arterial hypertension: a randomized, double-blind, placebo-controlled study (PATENT-1). Chest 142(4):1027A.

28. Ghofrani HA, Grimminger F, Grunig E, Huang Y, Jansa P, Jing ZC, Kilpatrick D, Langleben D, Rosenkranz S, Menezes F, Fritsch A, Nikkho S, Humbert M. Predictors of long-term outcomes in patients treated with riociguat for pulmonary arterial hypertension: data from the PATENT-2 open-label, randomised, long-term extension trial. Lancet Respir Med. 2016;4:361-71.

29. Ghofrani HA, Hoeper MM, Halank M, Meyer FJ, Staehler G, Behr J, Ewert R, Weimann G, Grimminger F. Riociguat for chronic thromboembolic pulmonary hypertension and pulmonary arterial hypertension: first long-term extension data from a phase II study. Am J Respir Crit Care Med. 2010;181:A6770.

30. Ghofrani HA, Galiè N, Grimminger F, Grunig E, Humbert M, Jing ZC, Keogh AM, Langleben D, Kilama MO, Fritsch A, Neuser D, Rubin LJ. Riociguat for the treatment of pulmonary arterial hypertension. N Engl J Med. 2013;369(4):330-40.

31. Ghofrani HA, D'Armini AM, Grimminger F, Hoeper MM, Jansa P, Kim NH, Mayer E, Simonneau G, Wilkins MR, Fritsch A, Neuser D, Weimann G, Wang C. Riociguat for the treatment of chronic thromboembolic pulmonary hypertension. N Engl J Med. 2013;369(4):319-29. 